\title{
USING A PULSE INTEGRATION TECHNIQUE FOR IMPROVEMENT OF RECEIVED SIGNAL POWER IN GROUND BASED SAR SYSTEM
}

\author{
M. Nekoee ${ }^{*}$, J. Amini $^{1}$ \\ ${ }^{1}$ Remote Sensing Division, School of Surveying and Geospatial Engineering College of Engineering, University of Tehran, Tehran, \\ Iran - (Mehran.Nekoee, Jamini)@ut.ac.ir
}

Commission III, WG III/2

KEY WORDS: Radar Remote Sensing, GB-SAR, Non-Coherent Pulse Integration, Radar Signal, PSLR

\begin{abstract}
:
Today, radar imaging with synthetic aperture has obtained an important place in radar remote sensing, in such a way that it is utilized in the variety of space borne, airborne and ground platforms to extract information from different desired surfaces. Among these, Ground Based Synthetic Aperture Radars (GB-SAR) has numerous applications such as space system calibration, change detection, target detection, surface displacement determination and condition monitoring. In general, in GB-SAR systems, given that the power of the transmitted signal is low, the received signal is also of low power, and subsequently the presence of target in the image is of lower probability. Therefore, in this research, a method is proposed to increase the received signal power based on pulse integration by a non-Coherent method which results in an increase in the Signal to Noise Ratio (SNR), thereby increasing the focusing power on the target. In this way, a large number of samples can be received in the data collection section and the gain can be therefore increased by integrating the number of received pulses. According to the $-3.22 \mathrm{~dB}$ Improvement of focusing evaluation criterion (PSLR), it is shown in this research that the accuracy of the target detection is increased. Finally, the effect of the number of pulses integrated on the noise presence and accuracy of the target detection is described.
\end{abstract}

\section{INTRODUCTION}

Utilizing microwaves, radar remote sensing has unique characteristics that have made this science a powerful tool in military, engineering, earth, space and atmosphere sciences. Among the characteristics of microwave based remote sensing systems, ability to collect information in day and night, ability to pass atmosphere and clouds, ability to penetrate some surfaces and capability of performing operations in all weather conditions can be mentioned (F. Ulaby et al., 2014). A Ground-Based SAR system consists of a radar sensor, a linear/nonlinear rail and a motor as the movement unit of the system (Bernardini et al., 2007). The radar sensor transmits signals by transmitter antenna and receives the echoes by receiver antenna. Resolution is described by two components in radar systems; first component is the resolution in line with radar range, which is dependent on the sent pulse band width which is determined during radar system design; the higher the modulation bandwidth the better the resolution in range direction (Carrara et al., 1995; Cumming et al., 2005). The second component is the resolution in line with azimuth or the radar system motion, which depends on the antenna size, distance to target and the sent wave length and to reach high resolution in azimuth direction, large antenna is required which is considered as the most important drawback of radar systems (Gregory L. Charvat 2014). In order to solve the low resolution in azimuth direction, synthetic aperture radar systems were developed (Cafforio, C., C. Pratti and F. Rocca, 1989, 1991, 1998). In these systems, a broad virtual beam is created using signal processing techniques to compensate for the small size of antenna and improve the azimuth direction resolution. Finally, in a synthetic aperture radar system, the azimuth direction resolution is dependent on the created synthetic aperture length, system to target distance, sent wave length and the antenna radiation angle relative to the motion of the sensor, in such a way that increasing the synthetic aperture length would result in better resolution. An interesting fact in synthetic aperture radar systems is that in contrary to radar systems, they will produce better azimuth direction resolution with smaller antenna size (Cumming et al., 2005). Data are collected in the form of a two dimensional matrix in these systems. The reason for this two dimensionality is the collection of data in two directions of range and azimuth, which would enter the signal processing part after collection and a complex radar image is produced at the end. In this paper, raw signals are supplied by a GB-SAR system has been developed in Microwave Remote Sensing Laboratory (MReSL) at the School of Surveying and Geospatial Engineering, University of Tehran, Tehran, Iran. The Range Migration Algorithm is used as Image Formation Processor (IFP) to generate Single-Look Complex (SLC) image.

\section{THE METHODOLOGY}

\subsection{Ground-Based SAR}

GB-SAR is a radar imaging system consisting of a sensor and a ground platform to move it. Resolution in a GB-SAR system is defined in two directions of range and azimuth. Like real aperture radar systems, range direction resolution is dependent on the sent pulse characteristics. This resolution for a not modulated rectangular pulse can be calculated from the following equation:

where $\quad \delta r=$ range resoluti

$$
\mathrm{C}=\text { light velocity }
$$

$\mathrm{T}=$ pulse duration

* Corresponding author 
But considering the signal processing theory, a modulated signal with the bandwidth of $\mathrm{B}$ can be compacted into a signal with the length of 1/B. The range direction resolution amount for the new modulated signal is:

$$
\delta r=\frac{C}{2 B}
$$

where $\quad \mathrm{B}=$ band width

One of the advantages of GB-SAR systems is better resolution in azimuth direction. Considering a point target at the scene center, the slant range to the target point changes while the sensor moves in its path. This hyperbolic form range change can be seen in the following figure. (Figure 1)

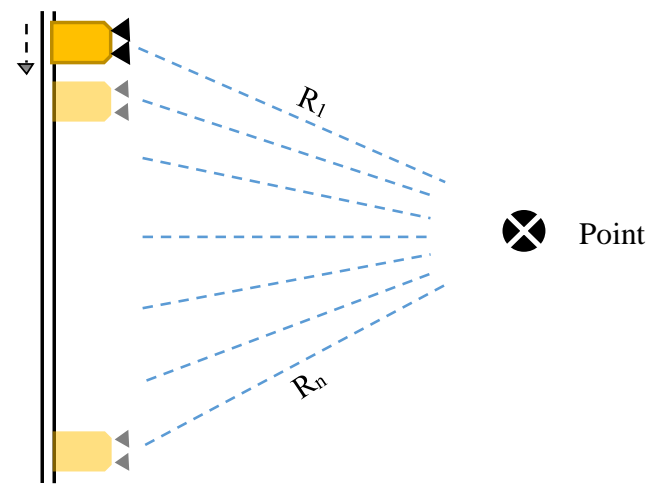

Figure 1. Different slant ranges in GB-SAR

This change in slant range has the following effect on the received signal which is shown in the below figure. (Figure 2)

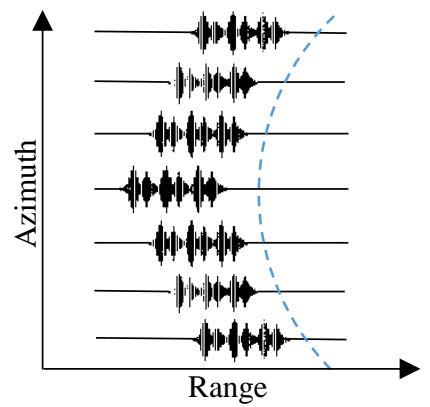

Figure 2. Different range profiles in GB-SAR

This very important feature states that the modulated signal can be compressed in the azimuth direction same as range direction. After compacting the azimuth direction signal, a much narrower beam is resulted which would cause the azimuth direction resolution. Using the following equation, the beam width can be calculated from the antenna size and the sent wavelength:

$$
\beta_{a} \cong 0.89 \frac{\lambda_{c}}{D}
$$

where $\quad \beta_{a}=$ beam width

$\lambda_{c}=$ wavelength of transmitted signal

$\mathrm{D}=$ antenna diameter

Finally, to obtain the azimuth direction resolution, the beam width must be multiplied with the sensor slant range to the point target. Therefore, the following equation holds for the azimuth direction resolution:

$$
\delta x=\beta_{a} \times R \cong 0.89 \frac{\lambda_{C}}{D} R
$$

where $\quad \delta x=$ azimuth resolution

$\beta_{a}=$ beam width

$R=$ slant range between target and sensor

This equation is stated in the following form after establishing the SAR geometry for the created synthetic aperture with the length of L:

$$
\delta x \cong \frac{\lambda_{C} K_{a} R}{2 L \sin \alpha_{d c}}
$$

$$
\begin{aligned}
& \text { where } \quad \delta x=\text { azimuth resolution } \\
& K_{a}=\text { beam width } \\
& \mathrm{L}=\text { synthetic length } \\
& \alpha_{d c}=\text { angle between transmitted signal and sensor } \\
& \quad \text { movement direction }
\end{aligned}
$$

\subsection{Radar Range Equation Applied to SAR}

As mentioned before, SAR system is formed by moving the radar sensor on a designed path. The large number of range profiles acquired from scene will improve radar performance in both azimuth resolution and maximum range. Therefore, the radar equation for a synthetic aperture radar is calculated as follows:

$$
R_{\text {max }}^{4}=\frac{N P_{a v e} G_{t x} A_{r x} \rho \sigma e^{2 \alpha R_{\max }}}{(4 \pi)^{2} K T_{0} B_{n} F_{n} L_{s} \tau F_{r}(S / N)_{1}}
$$

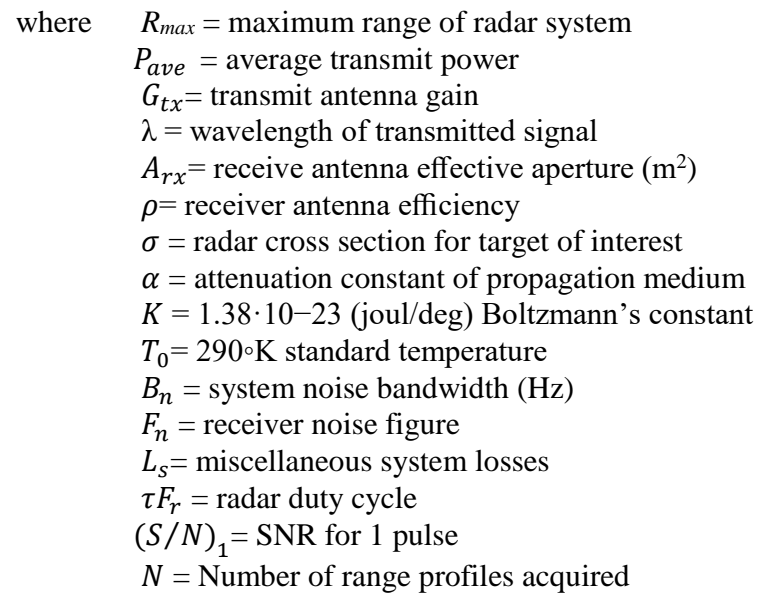

\subsection{Signal to Noise Ratio (SNR)}

Based on the signal theory, one of the important parameters of a radar system is the signal to noise ratio (SNR). This parameter describes the ability of the radar system to detect the target. According to the definition, the signal to noise ratio in radar describes the amount of difference in the level of power required at the receiver to detect a target with a specified cross-section at a specified distance from a specified radar.

In the radar systems, the sent signal is sent to the target with a specified power and then the distributed signal returns to the antenna from the target. This returned signal would change and 
becomes weaker depending on the radar - to - target distance, shape and material of the target, and therefore its power would decrease. Thus, the returned signal power is considered to calculate the signal to noise ration. The returned signal has the power obtained from the following equation:

$$
S=\frac{P_{a v e} G_{t} G_{r} \sigma \lambda^{2}}{(4 \pi)^{3} R^{4}}
$$

where $\quad G_{t}=$ transmitter gain

$$
G_{r}=\text { receiver gain }
$$

While the receiver antenna is collecting the returning signals, it also receives the effects that the environment has made on the signal. In addition to environment noises, the receiver itself as well as the hardware negatively affect the received signal. Overall, the total noise power in the receiver entrance is proportional to the system noise temperature and the receiver band width, and is calculated as follows:

$$
N=k T_{0} B_{n}
$$

According to the above mentioned, the signal to noise ratio is calculated from division of equations 7 and 8 :

$$
\frac{S}{N}=\frac{P_{a v e} G_{t} G_{r} \sigma \lambda^{2}}{(4 \pi)^{3} R^{4} k T_{0} B_{n}}
$$

\subsection{Non-Coherent Pulse Integration}

One of the ways to increase SNR is the radar pulse integration technique. In this technique, instead of analysing a returning pulse, sum of several returning pulses can be used to detect the target. Due to the fact that the signal processing in this study is in the field of radar, non-coherent integration method is used. In this method, only the amplitudes of returning pulses are integrated (Skolnik 2001, 2008). According to the following equation, the required signal to noise ratio (SNR) for detecting a pulse when $\mathrm{N}_{\text {eq }}$ pulses are integrated would be:

$$
(S / N)_{1}^{N_{e q}}=\frac{(S / N)_{1}^{1}}{N_{e q}}
$$

$$
\begin{array}{ll}
\text { where } \quad & (S / N)_{1}^{N_{e q}}=\mathrm{SNR} \text { for } N_{e q} \text { pulses } \\
& (S / N)_{1}^{1}=\mathrm{SNR} \text { for } 1 \text { pulses } \\
& N_{e q}=\text { number of pulses }
\end{array}
$$

Using this method the sional to noise ratio oain imnrovement is

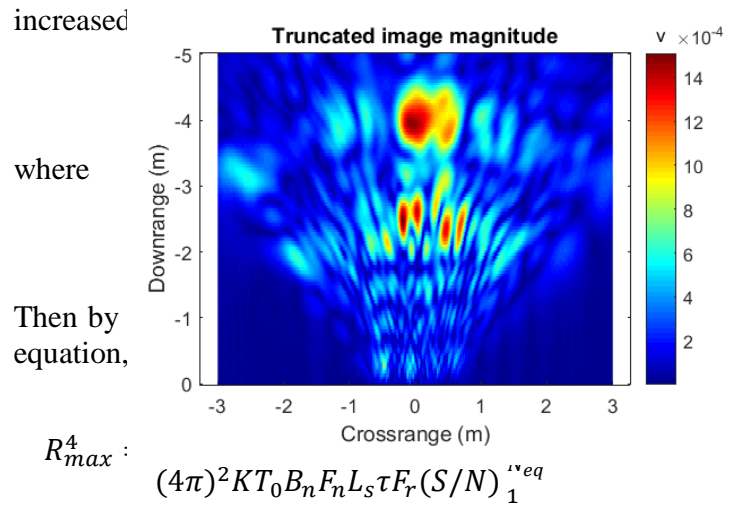

Finally, by combining the two equations of (10) and (12), the following equation for radar is obtained:

$$
R_{\text {max }}^{4}=\frac{N P_{\text {ave }} G_{t x} A_{r x} \rho \sigma e^{2 \alpha R_{\max }} N_{e q}}{(4 \pi)^{2} K T_{0} B_{n} F_{n} L_{s} \tau F_{r}(S / N)_{1}}
$$

where

$$
\begin{aligned}
& (S / N)_{1}^{N_{e q}}=\mathrm{SNR} \text { for } N_{e q} \text { pulses } \\
& (S / N)_{1}^{1}=\mathrm{SNR} \text { for } 1 \text { pulses } \\
& N_{e q}=\text { number of pulses }
\end{aligned}
$$

\section{EXPERIMENTAL RESULTS}

In this section, first the characteristics of GB-SAR system which used in this paper has been explained. These characteristics has been shown in Table 1 .

Table 1. MReSL GB-SAR characteristics

\begin{tabular}{|c|c|}
\hline Signal Type & FMCW \\
\hline Center frequency & $2.26 \mathrm{GHz}$ (S-band) \\
\hline Center wave length & $0.123 \mathrm{~m}$ \\
\hline Bandwidth & $330 \mathrm{MHz}$ \\
\hline Pulse duration & $22 \mathrm{msec}$ \\
\hline Range resolution & $0.454 \mathrm{~m}$ \\
\hline Azimuth resolution & $44 \mathrm{mrad}$ \\
\hline Aperture length & $1.4 \mathrm{~m}$ \\
\hline Number of range profiles & 140 \\
\hline
\end{tabular}

The purpose of this study is to proposed a method for improving the SAR images. In the proposed method, more pulses are used to detect the target. In the following, the results of the images compiled for the integration operation are presented taking into account the number of different pulses. The Non-Coherent integration operation has been implemented for the number of pulses 10, 20,30 and 40 and then each of the integrated samples entered the image forming algorithm. Finally, an integrated signal with real and imaginary values is formed for the range profile and was then prepared for entering the image formation algorithm. In the following, figure 3 shows SAR images using single pulse, 10, 30 and 40 integrated pulses in (Voltage) and (dB) scales. 


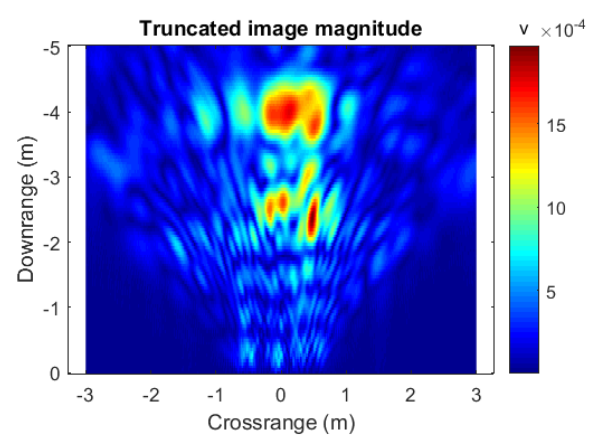

(b)

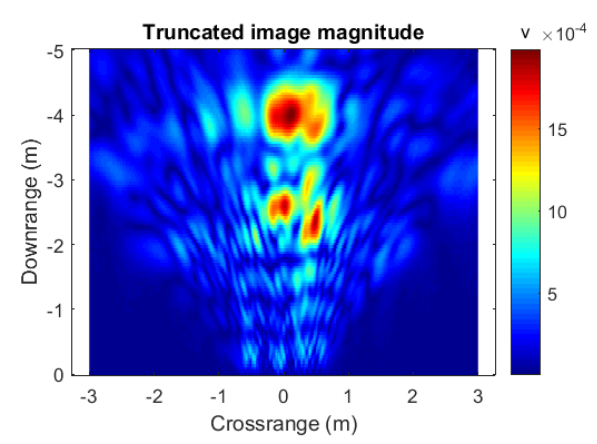

(c)

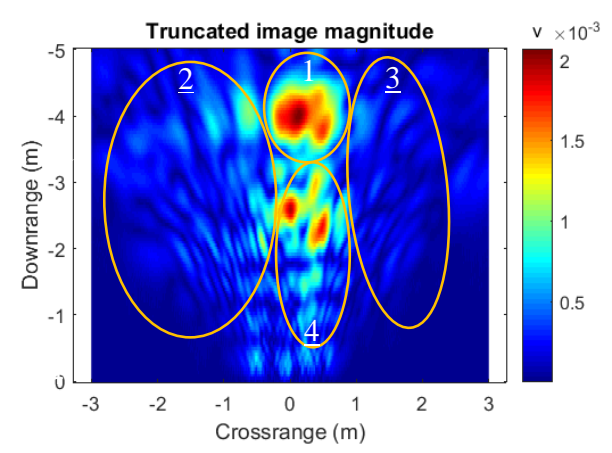

(d)

Figure 3. SAR image using

(a) 1 pulse (b) 10 integrated pulses (c) 30 integrated pulses (d) 40 integrated pulses in Voltage scale

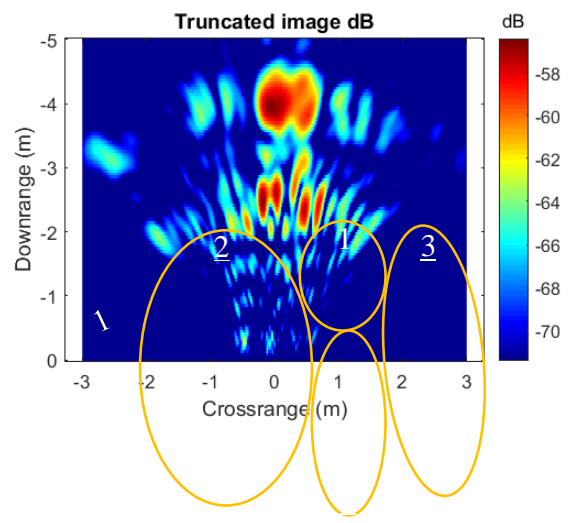

(a)

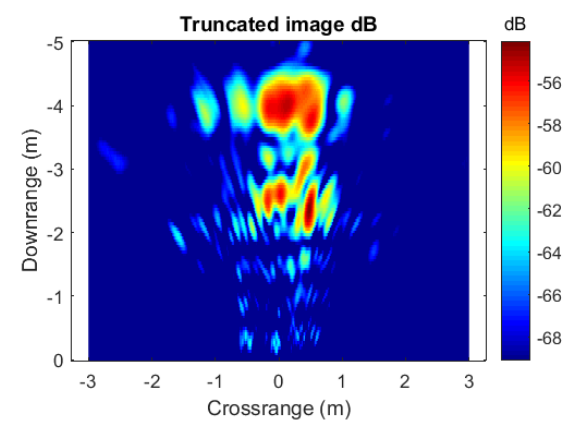

(b)

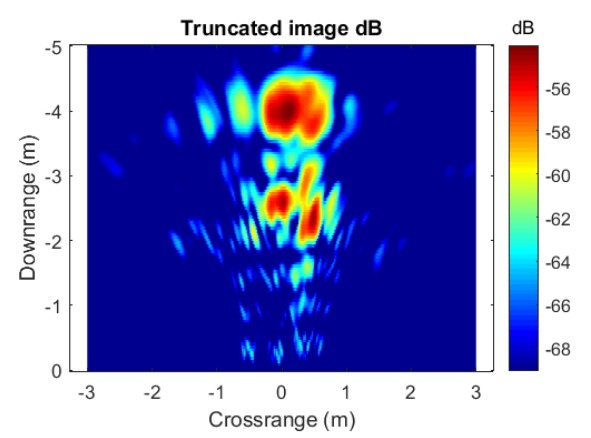

(c)

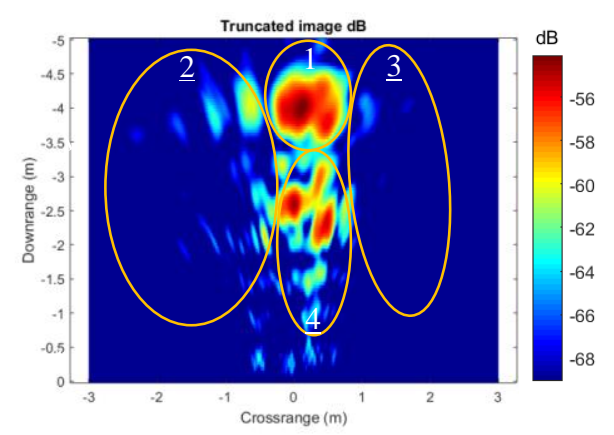

(d)

Figure 4. SAR image using

(a) 1 pulse (b) 10 integrated pulses (c) 30 integrated pulses (d) 40 integrated pulses in $\mathrm{dB}$ scale

After using pulse integration technique, according to the results, it can be concluded that in the zones 2,3 and 4, noise has been significantly reduced. This noise is the result of multidirectional targets. This reduction in noise will increase the signal-to-noise ratio and eventually lead to improved radar system. Zone 4 shows the noise due to interference of transmitted and received signals. It occurs because of inapplicable distance between transmitter and receiver antenna. This zone is also affected by pulse integration and as it is observable, the noise of interference has been reduced. In zone 1, after pulse integration, a decrease in side area intensity can be seen. This region represents the side lobe in the radar signal. Therefore, the lower the value, the greater the accuracy of the focusing operation. In this zone, after pulse integration, focusing on the target has been performed with greater accuracy and has promoted this portion of the signal that represents the main lobe (Peak value) in the radar signal. Regarding the contents stated in this section, the main lobe and 
side lobe values for each aggregation level are measured in radar signals. The PSLR criterion has been used to determine the accuracy of the target detection. This criterion expresses the accuracy of the target detection by the following equation:

$$
P S L R=10 \log _{10}\left(I_{s} / I_{m}\right) \quad(d B)
$$

where $\quad I_{S}=$ highest side lobe intensity

$I_{m}=$ main lobe intensity

Here first convert SAR image to $(\mathrm{dB})$ scale and then calculate PSLR criterion by the following equation:

$$
P S L R=M-S \quad(d B)
$$

where $\quad M=$ peak value of main lobe $\mathrm{S}=$ peak value of side lobe

The higher the PSLR, the more precisely the target is detected and closer to reality. The results of the measurements and the evaluation of the proposed method are shown in Table 2 .

Table 2. PSLR results $(\mathrm{dB})$

\begin{tabular}{|c|c|c|c|}
\hline $\begin{array}{c}\text { Number of pulses to } \\
\text { integration }\end{array}$ & $\begin{array}{c}\text { Peak } \\
\text { value } \\
(\mathrm{dB})\end{array}$ & $\begin{array}{c}\text { Side lobe } \\
\text { value } \\
(\mathrm{dB})\end{array}$ & PSLR \\
\hline 1 Pulses & -56.63 & -58.66 & -2.03 \\
10 Pulses & -53.31 & -57.54 & -4.23 \\
30 Pulses & -52.84 & -57.9 & -5.06 \\
40 Pulses & -52.66 & -57.91 & -5.25 \\
\hline
\end{tabular}

\section{CONCLUSIONS}

In this research, a method for increasing the received signal power in ground-based synthetic aperture radar systems was presented.The results suggested that pulse integration has a great effect on the noise present in the SAR image. By increasing the number of pulses, better results can be obtained by reducing the noise. Also, with the increase in the number of pulses, the focus power on the target and the power to set aside the wrong targets also increases. Given the calculated numerical values, it can be concluded that when 40 pulses are used to form an SAR image, the $-3.22 \mathrm{~dB}$ improvement is achieved by focusing precisely on the target. According to the results of this research, the importance of pulse integration for increasing the gain in low power radar systems can be realized and concluded that this method can play a role in improving radar images.

\section{ACKNOWLEDGMENT}

The experiments performed in this study was by means of the synthetic aperture radar system developed in Microwave Remote Sensing Laboratory (MReSL) at the School of Surveying and Geospatial Engineering, University of Tehran. Therefore, the authors would like to express their gratitude towards this laboratory and the people involved in the process of providing raw radar data from the target.

\section{REFERENCES}

Bernardini, G., Ricci, P., Coppi, F., 2007. A ground-based microwave interferometer with imaging capabilities for remote measurements of displacements. In: Proc. GALAHAD Workshop Within the $7^{\text {th }}$ Geomatic Week and the $3^{\text {rd }}$ International Geotelematics Fair (GlobalGeo), Barcelona, Spain, 20-23 February.

Cafforio, C., C. Pratti and F. Rocca, Full Resolution Focusing of SEASAT SAR EARSeL thomain, Proceedings of the 8Wave Number D-Images in the Freauency Symposium, Capri, Italy, 1998, pp. 336-355.

Cafforio, C., C. Pratti and F. Rocca, SAR Data Focusing Using Seismic Migration Techniques, IEEE Transactions on Aerospace and Electronic Systems, Vol. 27, No. 2, March 1991, pp. 194-206.

Carrara, W.G., Goodman, R.S., Majewski, R.M., 1995. Spotlight synthetic aperture radar: signal processing algorithms. Artech House, Boston.

Cumming, I.G., Wong, H., Digital Processi ng of Synthetic Aperture Radar Data: Algorithms and Implementation, Artech House, Norwood, 2005.

Charvat, G.L., Fenn, AJ., Perry, B.T., "The MIT IAP radar course: build a small radar system capable of sensing range, doppler and synthetic aperture radar (SAR) imaging," Atlanta, GA: IEEE Radar Conference, May 2012.

Gregory L. Charvat, Small and Short Range Radar Systems, CRC Press, 2014.

Rocca, F., C. Cafforio, and C. Pratti, Synthetic Aperture Radar: A New Application for Wave Equation Techniques, Geophysical Prospecting 37, 1989, pp, 809-830.

Skolnik, M., Introduction to Radar Systems. McGraw -Hill, New York, 2001.

Skolnik, M. Radar Handbook, 3rd ed.; The McGrawHill Companies, Inc.: New York, NY, USA, 2008.

Ulaby, F.T., et al., Microwave radar and radiometric remote sensing. Ann Arbor: University of Michigan Press, 2014. 